\title{
Orthodontically induced inflammatory root resorption: apical and cervical complications*
}

\author{
Dominique LUNARDI, Thibault BÉCAVIN, \\ Alain GAMBIEZ, Étienne DEVEAUX
}

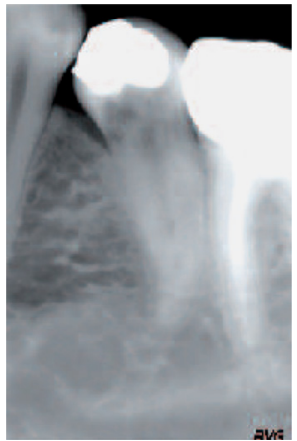

\begin{abstract}
External root resorption is a feared complication during orthodontic procedures. It can affect both the apex, but also the cervical zone of the roots subjected to orthodontic forces for tooth movement and can compromise the future of the involved teeth. The patient must be informed about the risks of resorption as a consequence of orthodontic treatment. The detection of resorptions can occur during and/or after the active phase of orthodontic treatment.

Knowledge of the clinical situations predisposing the patient to resorption is essential. The data are still insufficient to avoid them completely. Early and precise detection is essential, and the cone beam with its high resolution $3 D$ images, can compensate for the diagnostic inaccuracy of digital panoramic and periapical films. There are treatment solutions and their prognosis is correlated to early detection. Apical resorptions, generally not infected, can mostly be stopped by discontinuing orthodontic forces. Cervical resorptions, with a bacterial component, will require surgical intervention with curettage and restorative build-up of the resorbed area. Current research, particularly in genetics, is in the developmental stage. In the future, the conclusions of this research will allow orthodontists to more precisely target at risk patients so as to avoid these complications.
\end{abstract}

\section{KEY WORDS}

Inflammatory root resorptions,

Apical resorption,

Cervical resorption,

Orthodontic treatment,

Risk factors,

Cone beam. 


\section{INTRODUCTION}

Resorption is a clinical condition associated with a physiological or pathological process leading to a loss of dentin, cementum and/or bone structure ${ }^{7}$.

In orthodontics, therapeutic procedures mainly involve restructuring bone, periodontal ligaments and cementum ${ }^{1}$. All orthodontic treatment is accompanied by self-limiting and reversible micro-resorptions of root and cementum. Histologically, they are radiographically imperceptible surface resorptions, allowing and accompanying dental movements ${ }^{19}$. According to Andreasen et al. ${ }^{2}$, these surface resorptions are followed by spontaneous repair with the formation of new cementum. This is neither a physiological process nor a pathological process but instead a controlled orthodontic therapeutic process, as long as the dentin is not affected.

In certain cases, the phenomenon is no longer self-limiting: i.e. when the repair of the cementum is disturbed. The action of the odontoclasts reaches beyond the cementum to the underlying dentin. This is a pathological process because once the dentin is affected, it continues in an irreversible manner. These resorptions can affect the tooth at the apex as well as the neck. For non-infected apical resorption, even if the dentin cannot be reconstructed, newly formed cementum can cover the resorbed area ${ }^{19}$. Resorption shortens the root. The anatomy of cervical resorptions creates a predisposition to breeding bacteria at the resorption site. Because of its invasive nature ${ }^{13}$, this situation prevents new cementum from forming.

Given the importance of preventing these pathological resorptions, orthodontists must carefully take into account risk factors and conditions before initiating any treatment. By taking these precautions, orthodontic treatment can also be adapted to more difficult clinical situations.

If this preventive strategy fails and despite our efforts, invasive inflammatory resorptions appear, they should be detected early and accurately. A new diagnostic tool, the cone beam, already extensively used by endodontists, compensates for the diagnostic inaccuracy of panorexes and periapical radiography ${ }^{40}$. These two types of images do not clearly reveal resorption in all its configurations.

When this is the case, orthodontists and dentists must act in concert to set up a therapeutic strategy.

The earlier resorption is detected, the simpler the treatment solution is to safeguard teeth. A thorough followup during and after orthodontic treatment is therefore essential and it should be part of a long-term plan. In fact, pathological inflammatory resorptions show up long after the end of treatment and this is especially true for external cervical resorptions ${ }^{13}$. 


\section{HOW DO EXTERNAL PATHOLOGICAL INFLAMMATORY ROOT RESORPTIONS DEVELOP?}

Olivieri et al. ${ }^{29}$ explains that the development of external pathological inflammatory root resorptions results from the alteration or loss of the protective cementum layer due to inflammation. The cementum protects the underlying dentin from resorption and has the potential for repair $^{30}$. When a stimulus attacks the cementum and overwhelms its capacity for repair, dentin is exposed ${ }^{11,13}$.

Histologically, the dentin exposure to osteoclast precursor cells, originating in the periapical ligament and differentiating into odontoclasts, marks the starting point of the pathological process $^{11,13}$. In fact, only the cementum has the potential for repair, and as soon as the dentin is attacked and is in turn resorbed, the loss of root structure becomes irreversible ${ }^{19}$.

The forces exerted during orthodontic treatment can, in certain cases, lead to this type of irreversible resorption. The forces represent a potential mechanical stimulus for pressure on the cementum. Brezniak and Wasserstein $^{6}$, in 2002, suggested the use of terminology specific to orthodontics and spoke of orthodontically induced inflammatory root resorptions (OIIRR), emphasizing the importance of this phenomenon. In orthodontic literature, this is very often associated with the notion of apical resorption, but we should not forget that resorption can also involve the cervical area. Heithersay shows in one research article ${ }^{13}$, that the highest rate of cervical resorption involved patients who had earlier orthodontic treatment. The anatomical shape of the cervical resorption influences their development. These cervical resorptions are in effect subject, by way of the sulci, to a possible bacterial infestation promoting inflammation and hence the development of resorption, even if the movement forces are discontinued.

However, orthodontic forces are not the only etiology likely to cause disturbance in cementum repair and subsequently pathological root resorption.

It is important for the orthodontist to be aware of the possibility and nature of other possible causes of resorption. Therefore, they should also be listed as risk factors or additional etiological factors to orthodontically induced external root resorption. When diagnosing an external root resorption during or after orthodontic treatment, the practitioner can formulate a differential or complementary diagnosis.

Therefore, if Heithersay ${ }^{13}$ implicates orthodontic treatment in almost one fourth of recorded clinical cases of external cervical resorption, he also implicates traumas, internal bleaching, surgical and periodontal procedures, and other factors such as bruxism and delayed eruption of the dentition. 


\section{WHAT ARE THE PREDICTIVE FACTORS FOR THE APPEARANCE OF ROOT RESORPTIONS IN ORTHODONTICS?}

Mizrahi $^{27}$ reminds us that orthodontic treatment, like any other procedure of dentistry, exposes the practitioner to possible litigation.

An analysis of the predictive factors for the appearance of root resorption is indispensable before beginning orthodontic treatment and these factors should be listed in the informed consent form signed by the patient ${ }^{6}$.

In fact, certain patients are more predisposed than others to developing root resorption during orthodontic treatment ${ }^{11}$ and it is crucial to inform them. From a medico-legal perspective, Béry ${ }^{5}$ explains that the notion of risk is an integral part of patient information and that the practitioner should inform them about the known risks based on available scientific data.

For OIIRR, these risk factors are generally related to the genetic predisposition of the patients or to their general overall health. They can be local, depending on the patient's prior dental treatment. Finally, there are risk factors specific to the orthodontic treatment to the teeth that are involved.

\section{Genetic factors predisposing to resorption}

In some ethnic groups, we find the same susceptibility to orthodontically induced resorption ${ }^{16}$. According to Sameshima and Sinclair ${ }^{32}$, Hispanic patients are more susceptible to resorption than Asian patients. Further- more, Hartsfield ${ }^{11}$ explains that the activation of the osteoclasts (resorption cells) may be genetically linked. Iglesias-Linares et al. demonstrate that genetic variations involving the interleukine-1ß gene (implicated in the inflammatory process associated with orthodontic movements), may explain the appearance of certain external apical resorptions. All these articles present a variety of findings that argue in favor of developing this line of genetic research regarding OIIRR.

\section{Risk factors related to the general health of the patient}

Chronic asthma and certain allergies can increase the chances that OIIRR will develop.

Brezniak and Wasserstein ${ }^{6}$ report that patients with chronic asthma, whether treated or not, have a greater susceptibility to apical OIIRR on the upper molars. This occurs because asthma allows the inflammation of the sinuses to come into close contact with the apex of the maxillary molars and premolars.

\section{Risk factors related to prior dental procedures}

For young patients, prior traumatic incidents are common and it is not unusual for them to have a history of fractures, luxations and tooth loss followed by prosthetic replacement. In addition to any orthodontic treatment, 
the traumatic incidents alone could account for the appearance of inflammatory root absorptions. But, in cases of orthodontic treatment, these teeth that have already suffered trauma or even perhaps have already been resorbed will be all the more vulnerable to the forces that they will undergo. Brezniak and Wasserstein ${ }^{6}$ recommend waiting at least 3 months before exerting force on a transplanted or re-implanted tooth. However, in one study of the factors that predispose patients to cervical resorption, Heithersay ${ }^{13}$ remarks that the etiological association of orthodontic-trauma is relatively rare (3\% of the teeth studied). Nonetheless, together, orthodontics and trauma are frequently implicated in the etiology of resorption. Heithersay's findings might therefore be due the effective caution of orthodontists in applying force to move teeth that show signs of previous trauma.

Certain teeth whether traumatized or not, must be treated endodontically, and in the orthodontic literature, there are many debates about whether these filled teeth are more susceptible or less susceptible to resorption. There is no consensus about this question and in fact, researchers even arrive at contradictory findings. Mattison et al. ${ }^{26}$, and more recently Esteves et al. ${ }^{8}$ were unable to offer any evidence of significant differences between live and filled teeth. Mah et al. ${ }^{22}$ show that orthodontically induced apical resorptions are slightly greater for endodontically treated teeth even if the difference is not statistically significant, whereas Bender et al. ${ }^{4}$ arrive at the opposite conclusion. Based on our current understanding, we should use the same approach to vital and nonvital teeth in the context of orthodontic treatment, except if the endodontic treatment is associated with some previous trauma. Nonetheless, faced with all these contradictions, Iglesias-Linares et al. ${ }^{4}$ opened up new perspectives for research concerning endodontically treated teeth by focusing on the genetic component, especially interleukine-1ß.

For teeth that have undergone internal bleaching, the risk for resorption is described separately from any orthodontic treatment. Similar to traumas, in the case of orthodontic treatment, the risk for resorption of a tooth that has undergone an internal bleaching will be greater. However, this conclusion needs to be qualified since oxygenated water and the catalyst effect of heat due to friction were essentially implicated in the phenomenon of resorption after bleaching pulp-free teeth. The present use of sodium perborate mixed with water is a much safer procedure and less likely to induce resorption.

Other currently used therapeutic procedures that may have damaged the cementum of one or more teeth may be listed in the medical history of the patient and can represent areas at risk for resorption. Surgical procedures can, for example, because of trauma inflicted on neighboring teeth during manoeuvers for extraction (luxating and elevating with force), may be the cause for collateral trauma to cementum. Even if young patients are much less involved, periodontal detachment can also cause damage to the cementum and induce secondary external cervical resorption. 


\section{The risk factors associated with orthodontic treatment and the treated teeth}

All categories of teeth do not seem to have the same susceptibility to resorption. In his thesis, Makedonas ${ }^{23}$ refers to a number of studies and concludes that maxillary incisors, and more particularly lateral incisors are more frequently affected by orthodontically induced apical resorption. Apices that are either blunt or dropper-shaped present a greater degree of resorption compared to normally shaped apices. Next, he mentions the vulnerability of the lower incisors and the upper premolars.

The root anatomy must also be considered as a factor. Lavander and Malmgren $^{17}$ studied the relationship of the shape of the root to the incidence of apical resorption. Apices having thin "pipette-like" or blunt shapes had a greater degree of resorption than normally shaped apices.

There is a debate as to whether or not orthodontic forces alone cause root resorption. Should the applied forces be below a threshold so as to avoid resorption? Lopatiene and Dumbravaite $^{19}$ attempt to answer this question and agree on forces of 7 to $26 \mathrm{~g} / \mathrm{cm}^{2}$. Maltha et al. ${ }^{28}$ confirm that the magnitude of the force exerted is a decisive factor and that its intermittent application causes less damage than when it is continuous. Esteves et al. ${ }^{8}$ are in agreement and think that the use of intense force increases the likelihood of orthodontically induced resorption.

\section{When inflammatory resorption is induced by orthodontic treatment, when does it appear and what forms does it take?}

Resorption resulting from the force of orthodontic traction can occur on the apex as well as the cervical zone.

Apical resorption can appear during the active phase of orthodontic treatment and this is why the term resorption in orthodontics is often closely associated with the apex. Orthodontists diagnose them while performing routine radiographical examinations occurring periodically during the active phase of treatment. The 6-9 month period after beginning treatment seems to be important for screening and detecting apical resorption ${ }^{17,19}$. The detection of minor resorptions at this time would confirm that there is a high risk for subsequent aggravation of this phenomenon ${ }^{19}$.

As for cervical resorptions, they are generally diagnosed much later. Heithersay ${ }^{13}$ diagnosed them in patients whose orthodontic treatment had taken place 18 to 33 months earlier. Due to their late detection, these resorptions are not observed by the orthodontist earlier. In fact, rarely do patients subject themselves to regular, long-term post-treatment screenings. External cervical resorption differs also because of direct exposure to the oral environment and therefore to bacterial infestation. Due to this exposure, Heithersay ${ }^{12}$ is insistent about the invasive nature of these resorptions and describes their aggressive behavior. However, their 
progression is slow and their perforation of the pulp only occurs when there are serious lesions. The dentin probably contains an inhibitor for resorption ${ }^{39}$.

A system of classification for the various types of resorption makes it possible to make a precise diagnosis regarding their degree of severity.

For apical resorptions, the Levander and Malmgren system ${ }^{17}$ distinguishes four levels of apical lesion (Figs. 1 and 2):

-level 1: the resorption is minimal and simply leaves an irregular apical root contour.

-level 2: the resorption lesion is no greater than $2 \mathrm{~mm}$ on the hard tissues. The authors call it minor resorption.

-level 3: the resorption destroys up to the first third of the root. Therefore, the resorption is qualified as severe. -level 4: the resorption extends beyond the first third of the root length and is now considered extreme.

For external cervical resorption, the Heithersay system of classification distinguishes four levels of cervical lesion (Figs. 3 and 4):

-level 1: the resorption is a small invasive cervical lesion that presents a shallow dentinal erosion.

-level 2: the resorption lesion is very limited and penetrates the dentin close to the pulp chamber but does not extend as far as or only slightly onto the root dentin.

-level 3: the resorption lesion presents a deep penetration into the dentin up to the first third of the root.

-level 4: the resorption lesion is widely invasive and spreads apically beyond the first third of the coronal root.

\section{HOW TO DETECT OIIRR: INPUT FROM THE CONE BEAM}

Root resorption presents a clinically discreet symptomatology, that may be entirely absent, especially in the first stages of their development.

The clinical picture of an external cervical resorption can be limited to a simple sensation of discomfort with a pinkish cervical discoloration upon examination, whereas the clinical picture for apical resorption can be totally asymptomatic.

Because early detection is so essential, it calls for regular complementary radiographic examinations. The orthopantogram is currently used to conduct examinations during the course of treatment, but, even with periapical films, the diagnostic accuracy $^{40}$ of this radiographic examination is often limited.

Since the end of the '90s, a new tool has been available, known in the Anglophone world as $\mathrm{CBCT}^{20}$ or "cone beam computed tomography" scanner.

Scientific research that relies on CBCT for the study of orthodontically induced root resorption is increasingly evident in the literature. All these studies confirm the reliability and accuracy of the high resolution 3D images of the cone beam and 

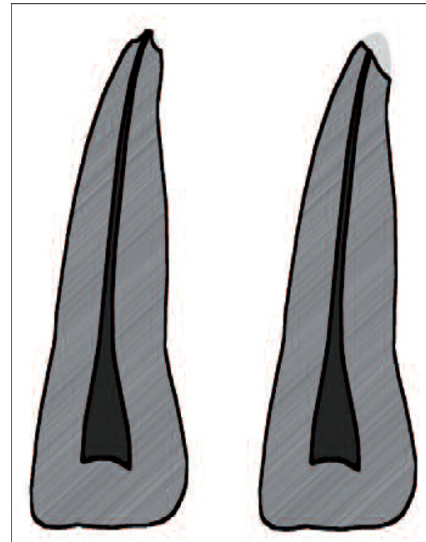

Niveau 1

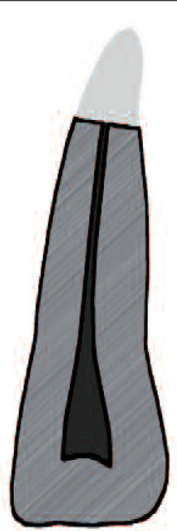

Niveau 3

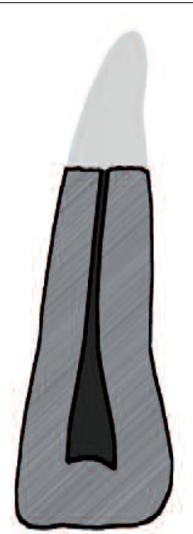

Niveau 4

Figure 1

The different levels of apical lesions laccording to Levander and Malmgren ${ }^{17}$ ).

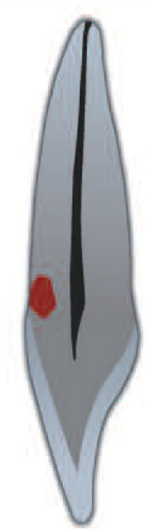

Niveau 1

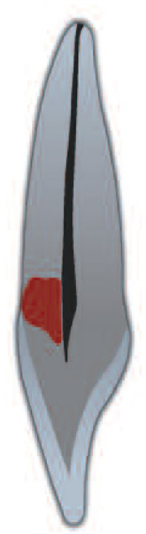

Niveau 2

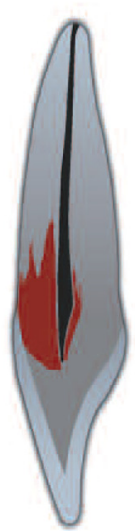

Niveau 3

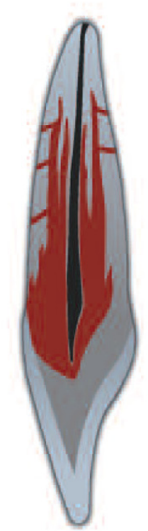

Niveau 4

Figure 3

The different levels of cervical lesions laccording to Heithersay ${ }^{13}$ ).

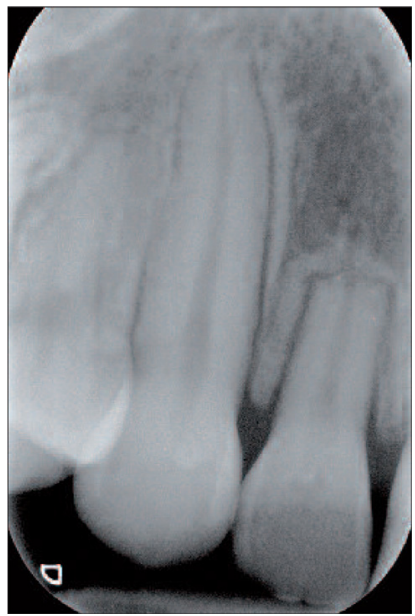

Figure 2

Orthodontically induced inflammatory root resorption on the upper right lateral incisor: extreme apical lesion, level 4 Levander and Malgren ${ }^{17}$.

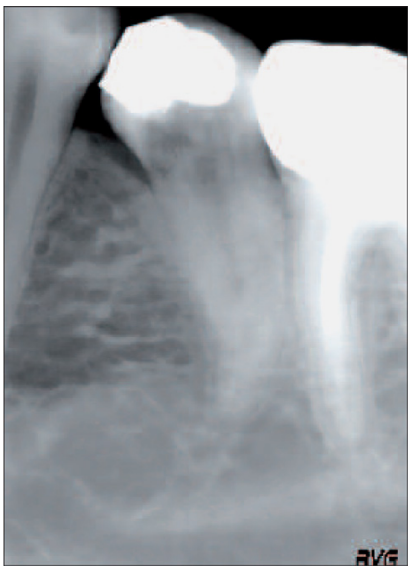

Figure 4

Orthodontically induced inflammatory root resorption on the upper left second premolar: deep cervical lesion, level 3 Heithersay ${ }^{13}$. 


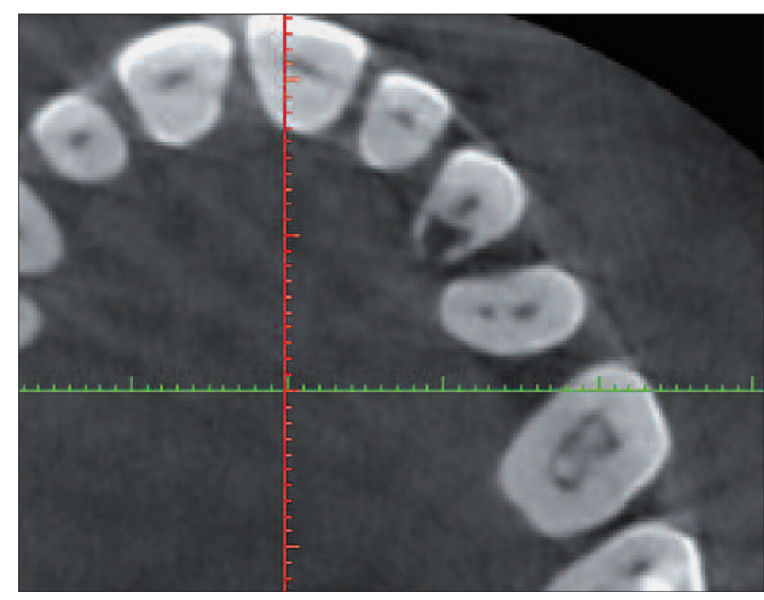

Figure 5

Cervical resorption detected with cone beam imaging on a maxillary canine (transversal cut).

show how valuable it is as a tool for detecting resorption $3,21,24,28$.

The $\mathrm{HAS}^{10}$ in France (similar to the FDA) states that there is "a spatial resolution and reliability comparable to that of a scanner" (Fig. 5). The HAS continues "the accuracy and reliability of the measurements of CBCT makes its use in the dentomaxillo-facial orthopedic discipline appropriate since the delivered radiation dose is less than that of a scanner".
The HAS report evaluates the benefits of new technology as they pertain to each dental discipline.

In orthodontics, the conclusion of the report indicates that, for now, CBCT cannot be used in place of conventional teleradiography for diagnostic purposes. However, in endodontics, the report mentions that in the interest of accuracy the cone beam should be used when information gathered from clinical examinations and radiography do not provide sufficient data for diagnosis and therefore a tridimensional image is indispensable. This statement applies perfectly to the detection and diagnosis of root resorptions (Fig. 6a to 6c).

Recently, Wang et al. ${ }^{38}$ studied in vivo the accuracy of volumetric measurements of the cone beam on a sampling of 27 maxillary and mandibular premolars. The authors conclude that the use of CBCT in the detection of orthodontically induced root resorption is an obvious possibility. Lund $^{20}$ demonstrates the existence of resorption on both palatal and lingual surfaces. He also specifies that they would not have been detected with conventional radiography.

\section{WHAT ARE THE THERAPEUTIC SOLUTIONS?}

Therapeutic solutions are closely related to both the location of the resorption (apical or cervical), and to their stage of development (especially for cervical resorption).

Throughout orthodontic treatment, the practitioner will most often encounter apical resorption. In fact, it is especially during the active phase of orthodontic treatment that resorption appears. To the extent that routine radiographic screening is performed, the orthodontist is more likely to detect them at the initial point of their development. This is why even if severe cases are detected, they are relatively uncommon ${ }^{19}$. Sehr et al. ${ }^{33}$ examined a little more than 3000 patients who underwent multi-bracket treatment between 1991 and 2010: 

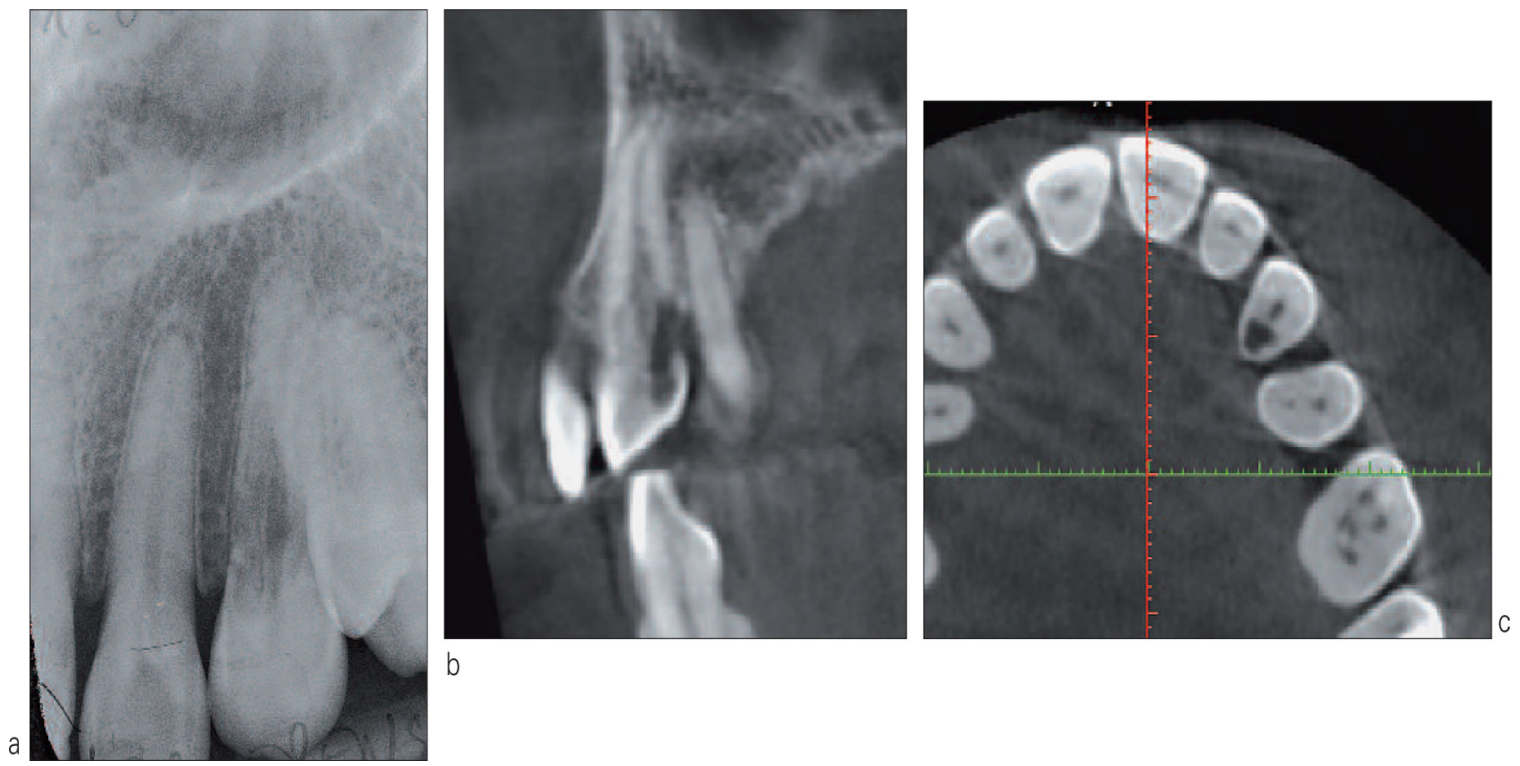

b

.Figures 6 a to $6 c$

a: periapical view with suspicion of orthodontically induced external root resorption on a left upper canine;

b: saggital cut with cone beam imaging: confirmation and precise imaging of cervical resorption;

c: transverse cut with cone beam imaging: confirmation and precise imaging of cervical resorption.

severe apical resorptions were detected in 16 patients. Makedonas ${ }^{23}$ confirms that the literature only comes up with an insignificant percentage of moderate to severe cases of resorption.

When the first radiographic signs appear, it is advisable to temporarily interrupt orthodontic treatment for a period of 3 months $^{31}$. In a survey of recent literature, Walker ${ }^{37}$ says that stopping treatment for 2 to 3 months makes it possible to completely reduce the progression of root resorption. Tirpuwabhrut et al. ${ }^{34}$ adopt the solution of stopping treatment and confirm that in instances of orthodontically induced resorption, the practitioner should, in this case, follow the protocol essentially based on eliminating the causal factor, i.e. orthodontic displacements. The Norwegian authors continue to state that in the absence of infection (in other words pulpal necrosis), endodontic treatment is absolutely useless and would even be contra-indicated since the elimination of the pulp of a tooth during the resorptive process does not restrict the development of resorption. Routine clinical examination of the pulpal vitality of the treated teeth is however essential, because even if the pulpal disturbances are minimal when IOORR occurs ${ }^{34}$, the practitioner should never exclude the possibility of necrosis. Other authors recommend the use of ultrasound or anti-inflammatory medication ${ }^{35}$. There is not a plethora of research in this area and it is difficult to determine the best protocol. Once the resorption process is stabilized, the practitioner can resume orthodontic treatment and cautiously monitor the results by performing very precise 


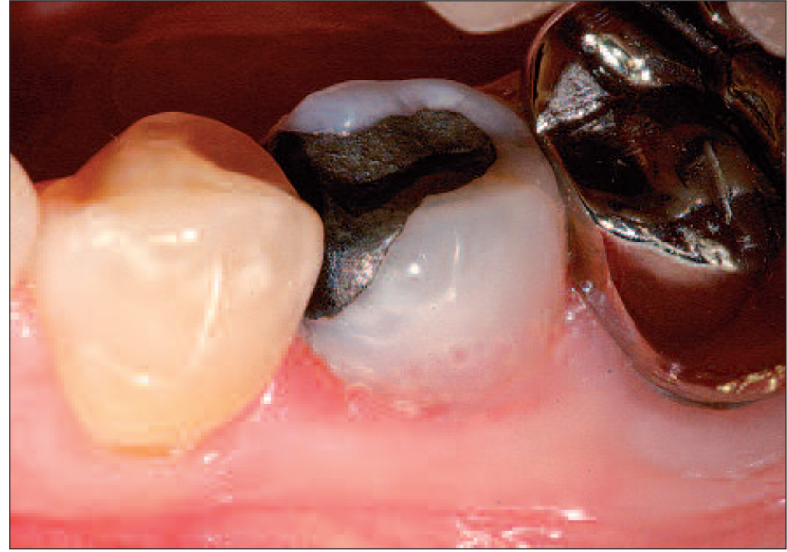

Figure 7

Pinkish cervical discoloration indicating the presence of an underlying external root resorption, on the left lower second premolar.

radiographic examinations, particularly by the use of CBCT.

External cervical resorption is often detected after orthodontic treatment is completed. According to Heithersay $^{13}$, the detection can occur from $1 \frac{112}{2}$ years to 33 years after orthodontic treatment. Therefore, most frequently either a dentist or an endodontist will make the diagnosis and choose the therapeutic treatment for resorption. Based on their location, external cervical resorption will be in direct communication with the oral environment and hence will be rapidly invaded by the bacterial flora. Because of this infestation, Heithersay ${ }^{14}$ emphasizes their invasive nature. This characteristic of their symptomatology differentiates them from apical resorptions, especially regarding their development and treatment. As soon as the first signs are detected either clinically or radiologically, the practitioner should take an interventionist therapeutic approach. Although the diagnosis is often made radiologically, clinically a pink spot can appear in the cervical area. This pinkish discoloration signals the presence of very vascularized granular tissue under both the enamel and the dentin that are eroded by resorption ${ }^{14}$ (Fig. 7). The intervention will therefore consist of (sometimes after lifting the periodontal flap) a total elimination of the granulomatous tissue, followed by a reconstruction of the residual cavity of the resorption with either glass ionomer or composite cement (Mineral Trioxide Aggregate or Biodentine ${ }^{T M}$ ) (Fig. 8 a to $8 d$ ). This therapeutic approach has four main objectives: to stop the process of resorption, to restore the lost structure, to prevent a recurrence and finally to preserve esthetics ${ }^{30}$. Vinothkumar et al. ${ }^{36}$ present a clinical case where a Heithersay level 2 resorption is treated surgically, and then restored using the reverse sandwich technique combining composite and modified glass ionomere cement.

The decision for intervention depends on the extent of the lesion's development and its placement on the Heithersay scale ${ }^{14}$. For levels 1 and 2 of resorption, Heithersay indicates that intervention is often the right prognosis since the pulp is still protected both by outer and inner dentinal walls. For level 3, the clinical condition is more complicated but intervention is still indicated ${ }^{9}$. However, when the cervical lesion is level 4 , as long as it remains asymptomatic, Heithersay advises that the practitioner withhold treatment (Fig. 9 $a$ and $9 \mathrm{~b})$. In fact, intervention at this stage runs the risk of fracturing and therapeutic failure that would require removal of the tooth. When level 4 is accompanied by all signs and symptoms, the tooth must be removed. 


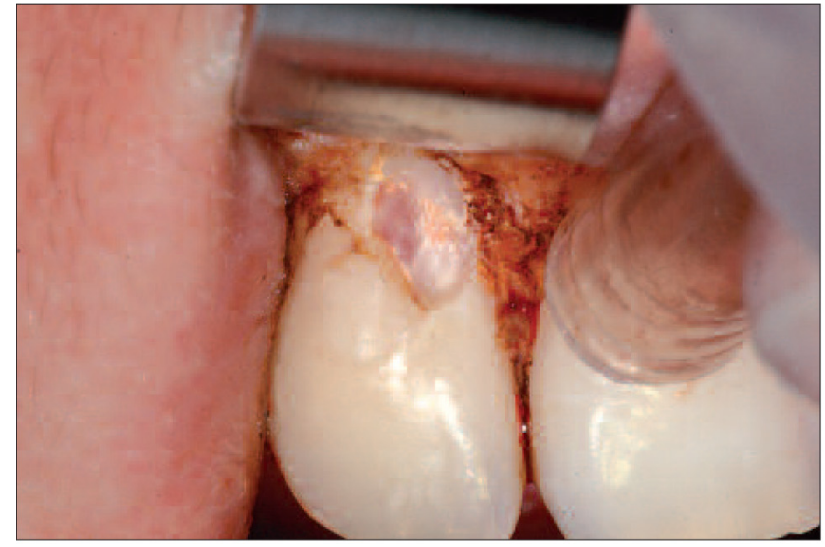

a

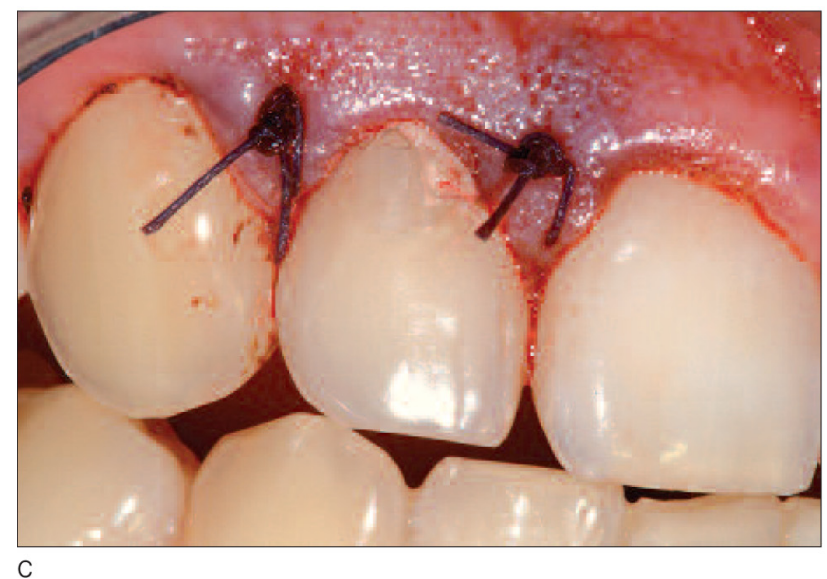

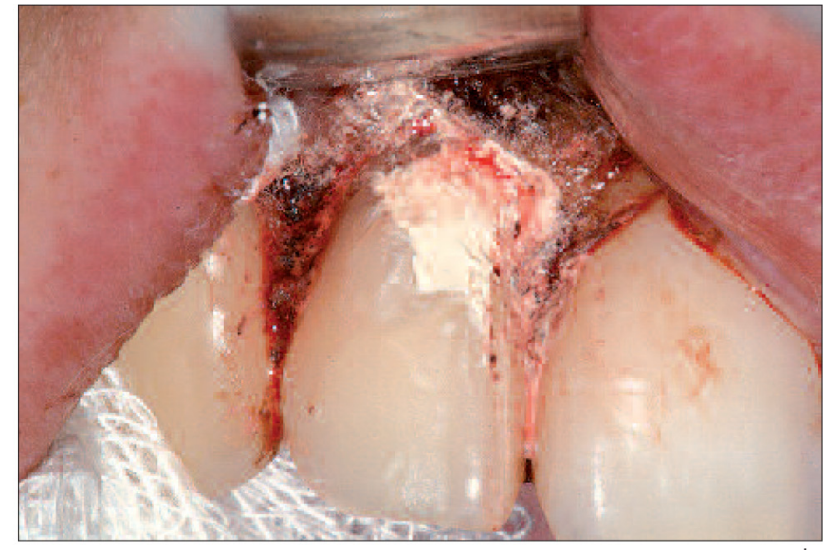

b

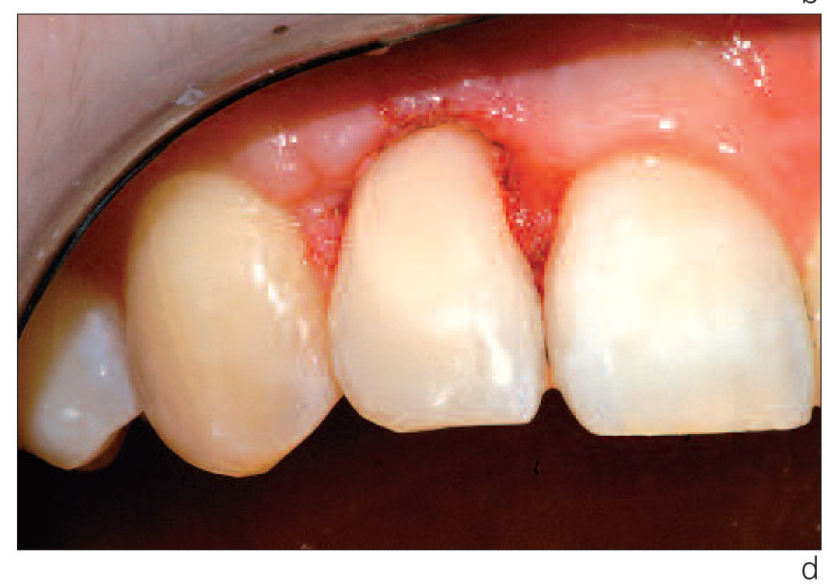

Figures 8 a to $8 d$

a: residual cavity of resorption after eliminating pathological tissue, on the upper right lateral incisor;

b: restoration with Biodentine ${ }^{T M}$ cement;

$c$ : repositioning of the periodontal flap and sutures;

d: cervico-coronary restoration using the sandwich technique with composite cement.

\section{CONCLUSION}

The risk of orthodontically induced root resorption is a proven fact, but in most cases, it can be controlled. Most importantly, the patient should be informed about the inherent risk of resorption. "It is better to inform beforehand than to be sorry afterwards when it is too late" 6 . Therefore, it is important to screen for resorption in every case but even more so for high risk cases during both the active phase of treatment as well as after the end of treatment. This monitoring must include both the apical and the cervical zones of the teeth subject to orthodontic forces. If there is suspicion of resorption, the cone beam is a very precise diagnostic reference point. It makes it possible to determine the anatomical context, as well 
Figures $9 a$ and $9 b$ a: orthodontically induced external cervical resorption: wide invasive lesion, Heithersay ${ }^{17}$ level 4, on the left lower second premolar; $b$ : corresponding periapical view.
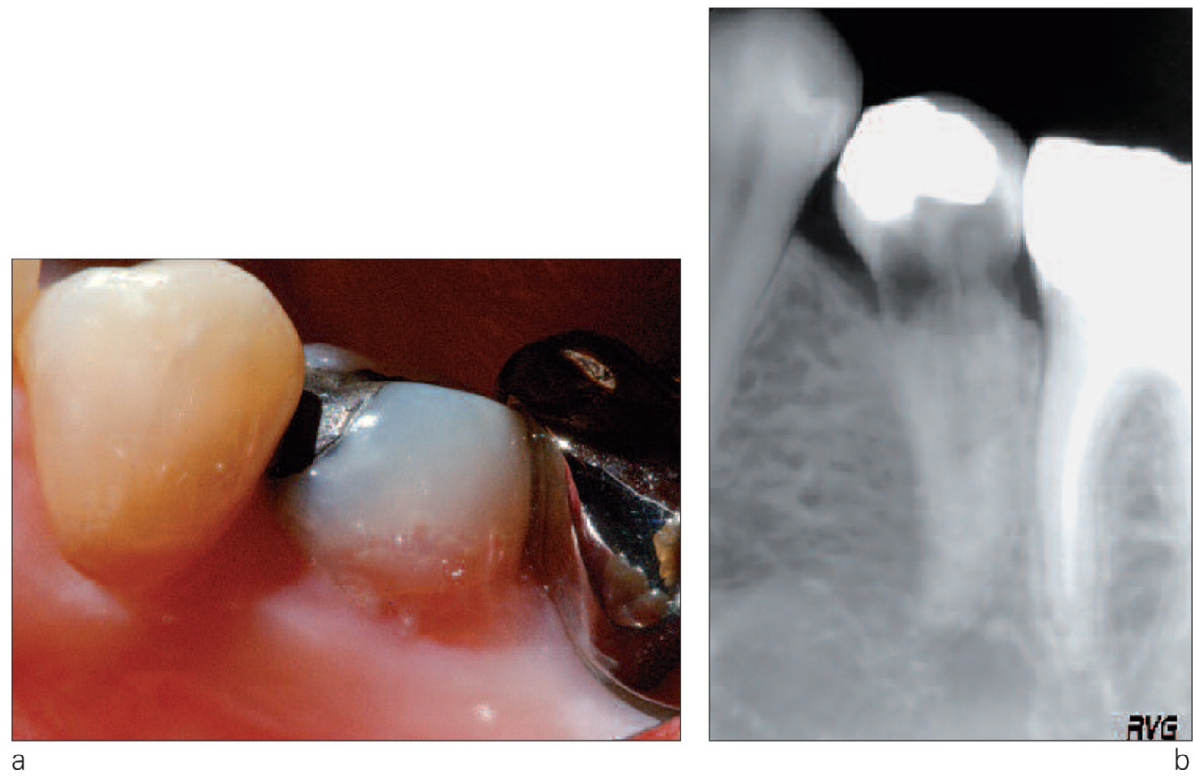

as the extent and severity of the resorption. Starting with an accurate diagnosis makes the therapeutic management easier for the practitioner.

Conservative therapeutic treatment exists and resorting to extraction is still the exception. For the orthodontist, it is essential to temporarily or even indefinitely stop treatment in cases of apical resorption in order to halt the process of resorption. In cases of cervical resorption, the orthodontist should intervene as soon as resorption is detected given its invasive and evolving nature when directly exposed to the oral environment. Orthodontists, dentists and endodontists will have to collaborate in order to improve the prognosis of the most difficult clinical situations.
Informing the patients and obtaining their informed consent, a thorough medical history and an evaluation of risk, routine screening and early and accurate detection with the use of cone beam technology, and finally, collaborative and multidisciplinary management of therapeutic treatments therefore represent the key elements for the management of orthodontically induced apical and cervical resorptions. However, presently, we are still unable to completely eliminate the occurrence of OllRR even though Brezniak and Wasserstein ${ }^{6}$ optimistically hope that future research will make it possible.

\section{Acknowledgements}

We wish to thank Rémy Faucomprez for diagrams that appear in this article.

\section{REFERENCES}

1. Abuara A. Biomechanical aspects of external root résorption in orthodontic therapy. Med Oral Patol Oral Cir Bucal 2007;12(8):E610-3. 
2. Andreasen JO, Andreasen FM, Andersson L. Textbook and color atlas of traumatic injuries to the teeth. $4^{\text {th }}$ ed. Copenhagen: Munksgaard, 2007.

3. Baysal A, Karadede I, Hekimoglu S, Ucar F, Ozer T, Veli I, Uysal T. Evaluation of root resorption following rapid maxillary expansion using cone-beam computed tomography. Angle Orthod 2012;82(3):488-94.

4. Bender IB, Byers MR, Mori K. Periapical replacement resorption of permanent, vital, endodontically treated incisors after orthodontic movement: report of two cases. $J$ Endod 1997;23(12):768-73.

5. Bery A. Résorptions radiculaires et responsabilité. Rev Orthop Dento Faciale 007:41:349-54.

6. Brezniak N, Wassertein A. Orthodontically induced inflammatory root resorption. Part II: the clinical aspects. Angle Orthod 2002;72(2):180-4.

7. Contemporary terminology for endodontists. Glossary of endodontic terms. American Association of Endodontists. 2003.

8. Esteves T, Ramos AL, Pereira CM, Hidalgo MM. Orthodontic root resorption of endodontically treated teeth. J Endod 2007;33(2):119-22.

9. Estevez R, Aranguren J, Escorial A, de Gregorio C, De La Torre F, Vera J, Cisneros R. Invasive cervical resorption Class III in a maxillary central incisor: diagnosis and follow-up by means of cone-beam computed tomography. J Endod 2010;36(12):2012-4.

10. Haute Autorité de Santé. Tomographie volumique à faisceau conique de la face. Texte court du rapport d'évaluation technologique, décembre 2009.

11. Hartsfield JK Jr. Pathway in external apical root resorption associated with orthodontia. Orthod Craniofac Res 2009;12(3):236-42.

12. Heithersay GS. Clinical, radiologic and histopathologic features of invasive cervical résorption. Quintessence Int. 1999;30(1):27-37.

13. Heithersay GS. Invasive cervical resorption: an analysis of potential predisposing factors. Quintessence Int. 1999;30(2):83-95.

14. Heithersay GS. Management of tooth resorption. Australian Dental J 2007;52(1 Suppl):S105-21.

15. Iglesias-Linares A, Yañez-Vico RM, Ortiz-Ariza E, Ballesta S, Mendoza-Mendoza A, Perea E, Solano-Reina E. Postorthodontic external root resorption in root-filled teeth is influenced by interleukin-1ß polymorphism. J Endod 2012;38(8):283-7.

16. Le Norcy $E$, Lautrou A, Le Goff C. Facteurs affectant la résorption radiculaire associée aux traitements d'orthodontie. International Orthodontics 2005;3(2):129-40.

17. Levander $E$, Malgrem $O$. Evaluation of the risk of root resorption during orthodontic treatment: a study of upper incisors. Eur J Orthod 1988;10(1):30-8.

18. Liu Z, Xu J, E L, Wang D. Ultrasound enhances the healing of orthodontically induced root resorption in rats. Angle Orthod 2012;82(1):48-55.

19. Lopatiene K, Dumbravaite A. Risk factors of root resorption after orthodontic treatment. Stomatologija, Baltic Dental Maxillofacial J 2008;10(3):89-95.

20. Lund $\mathrm{H}$. Cone beam computed tomography in evaluations of some side effects of orthodontic treatment. Swed Dent J (Suppl). 2011;219:4-78.

21. Lund H, Gröndahl K, Hansen K, Gröndahl HG. Apical root resorption during orthodontic treatment. A prospective study using cone beam CT. Angle Orthod 2012; 82(3):480-7.

22. Mah R, Holland G.R, Pehowich E. Periapical changes after orthodontic movement of root-filled ferret canines. J Endod 1996;22(6):298-303.

23. Makedonas DT. Orthodontically induced root resorption: a clinical and radiographic survey. University of Gothenburg. Sweden 2012 [Thesis].

24. Makedonas D, Lund H, Gröndahl K, Hansen K. Root resorption diagnosed with cone beam computed tomography after 6 months of orthodontic treatment with fixed appliance and the relation to risk factors. Angle Orthod 2012;82(2):196-201. 
25. Maltha JC, van Leeuwen EJ, Dijkman GE, Kuijpers-Jagtman AM. Incidence and severity of root resorption in orthodontically moved premolars in dogs. Orthod Craniofac Res 2004;7(2):11521.

26. Mattison GD, Delivanis HP, Delivanis PD, Johns PI. Orthodontic root resorption of vital and endodontically treated teeth. J Endod 1984;10(8):354-8.

27. Mizrahi E. Risk management in clinical practice. Part 7. Dentolegal aspects of orthodontic practice. Br Dent J 2010;209(8):381-90.

28. Oberoi S, Knueppel S. Three-dimensional assessment of impacted canines and root resorption using cone beam computed tomography. Oral Surg Oral Med Oral Pathol Oral Radiol 2012;113(2):260-7.

29. Olivieri JG, Duran Sindreu F, Mercadé M, Pérez N, Roig M. Treatment of a perforating inflammatory external root resorption with mineral trioxide aggregate and histologic examination after extraction. J Endod 2012;38(7):1007-11.

30. Patel S, Kanagasingam S, Pitt Ford T. External cervical resorption: a review. J Endod 2009;35(5):616-25.

31. Samadet V. Entretien avec Pierre Machtou : à propos des résorptions radiculaires liées au traitement d'orthopédie dento-faciale. Rev Orthop Dento Faciale. 2007;41:253-62.

32. Sameshima GT, Sinclair PM. Predicting and preventing root resorption: Part I. Diagnostic factors. Am J Orthod Dentofac Orthop 2001;119(5):505-10.

33. Sehr K, Bock NC, Serbesis C, Hönemann M, Ruf S. Severe external apical root resorption: local cause or genetic predisposition? J Orofac Orthop 2011;72(4):321-31.

34. Tripuwabhrut $P$, Brudvik P, Fristad I, Rethnam S. Experimental orthodontic tooth movement and extensive root resorption: periodontal and pulpal changes. Eur J Oral Sci 2010;118(6):596-603.

35. Villa PA, Oberti G, Moncada CA, Vasseur O, Jaramillo A, Tobón D, Agudelo JA. Pulpdentine complex changes and root resorption during intrusive orthodontic tooth movement in patients prescribed nabumetone. J Endod 2005;31(1):61-6.

36. Vinothkumar TS, Tamilselvi R, Kandaswamy D. Reverse sandwich restoration for the management of invasive cervical resorption: a case report. J Endod 2011;37(5):706-10.

37. Walker S. Root resorption during orthodontic treatment. Evid Based Dent 2010; 11(3):88.

38. Wang Y, He S, Yu L, Chen S. Accuracy of volumetric measurement of teeth in vivo based on cone beam computer tomography. Orthod Craniofac Res 2011;14(4):206-12.

39. Wedenberg C. Evidence for a dentin-derived inhibitor of macrophage spreading. Scand J Dent Res 1987;95(5):381-8.

40. Yu L, He S, Chen S. [Diagnostic accuracy of orthopantomogram and periapical film in evaluating root resorption associated with orthodontic force]. Hua Xi Kou Qiang Yi Xue Za Zhi 2012;30(2):169-72. 